\begin{tabular}{|c|c|}
\hline Title & Development of a Numerical Simulation Method for the Magnetic Separation of Magnetic Particles \\
\hline Author(s) & Noguchi, So; Kim, SeokBeom \\
\hline Citation & $\begin{array}{l}\text { IEEE Transactions on Magnetics, 47(5), 898-901 } \\
\text { https://doi.org/10.1109/ MA G.2010.2072911 }\end{array}$ \\
\hline Issue Date & $2011-05$ \\
\hline Doc URL & http:/hdl.handle.net/2115/45423 \\
\hline Rights & $\begin{array}{l}\text { (c) } 2011 \text { IEEE. Personal use of this material is permitted. Permission from IEEE must be obtained for all other uses, in } \\
\text { any current or future media, including reprinting/republishing this material for advertising or promotional purposes, } \\
\text { creating new collective works, for resale or redistribution to servers or lists, or reuse of any copyrighted component of } \\
\text { this work in other works. }\end{array}$ \\
\hline Tyре & article (author version) \\
\hline File Information & ToM47-5_898-901.pdf \\
\hline
\end{tabular}

Instructions for use 


\title{
Development of a Numerical Simulation Method for the Magnetic Separation of Magnetic Particles
}

\author{
So Noguchi ${ }^{1}$ and SeokBeom Kim ${ }^{2}$ \\ ${ }^{1}$ Graduate School of Information Science and Technology, Hokkaido University, \\ Kita 14 Nishi 9, Kita-ku, Sapporo 060-0814, Japan \\ ${ }^{2}$ Department of Electrical and Electronic Engineering, Okayama University, \\ 3-1-1 Tsushima-naka, Kita-ku, Okayama 700-8530, Japan
}

\begin{abstract}
Magnetic separation is a very useful tool in medicine manufacturing, sludge disposal and we have developed the magnetic chromatography system, which separates the magnetic particles or the ions from fluid due to its strong magnetic field gradients in the very small flow channel. There are many fine ferromagnetic wires on the wall of the developed magnetic column. A superconducting magnet applies a strong magnetic field to the magnetic column, and fine ferromagnetic wires make strong magnetic field gradients. It was, however, impossible to accurately evaluate the performance of the developed magnetic column due to the absence of a good simulation method. In order to enhance the accuracy of the simulation it is necessary to couple the fluid dynamics simulation with the magnetic field simulation. Therefore, we have developed a simulation code dealing with the fluid dynamics, solving Navier-Stokes, control volume, and magnetic field equations simultaneously, and compared the simulation result with the experimental one to verify the validity.
\end{abstract}

Index Terms—Magnetic separation, magnetic chromatography, ferrohydrodynamics, numerical simulation.

\section{INTRODUCTION}

$\mathrm{M}$ AGNETIC SEPARATION is a very useful system for the fine magnetic particle or/and ion separation due to the strong magnetic field gradient in a very small flow channel. Many systems with the magnetic separation have been investigated and developed, e.g. for medicine manufacturing, sludge disposal, and drag delivery [1]-[5]. We have developed a magnetic chromatography system prototype with a novel magnetic column, which uses strong magnetic field gradients for separating magnetic particles with different magnetic susceptibilities in a small flow channel [6], [7]. Fine ferromagnetic wires are located on the wall of the flow channel inside the magnetic column. Therefore, the magnetic particles with large magnetic susceptibility are extracted in the radial direction of the flow channel and concentrated around the channel wall, and the flow velocity close to the channel wall is almost zero. The magnetic particles with small magnetic susceptibility go through the flow channel with less attraction for the channel wall. Thus, the distribution of the magnetic particle concentration in radial direction would be different and the exhaustion time must be also different.

In order to evaluate the performance of the magnetic column, a simulation method to analyze the fluid flow and the magnetic field is required. However, the design of magnetic separation system was based on the computation of only the magnetic field gradients, not considering the fluid flow [3], [5], [8]-[12]. On the other hand, the fluid flow was simulated based on the 2D fluid dynamics analysis with a simplified magnetic field analysis [6], [7]. The magnetic field analysis is done only once before simulating the time-varying fluid flow, though the time-varying concentration of the magnetic particles affects the magnetic field distribution and its gradients. That is, it is impossible to accurately evaluate the

Manuscript received May 31, 2010. Corresponding author: S. Noguchi (e-mail: noguchi@ssi.ist.hokudai.ac.jp).

Digital Object Identifier inserted by IEEE performance of the magnetic column and to accurately simulate the ferrohydrodynamics due to the absence of good analysis methods. Hence, an analysis method considering both ferrohydrodynamics and magnetic field equations is strongly desired.

We have developed the simulation code to analyze the magnetic fluid flow, taking into account Navier-Stokes, control volume and magnetic field equations simultaneously. The Navier-Stokes equation represents the flow of the magnetic fluid. The concentration of the magnetic particles considering its diffusion is given by the control volume equation. The magnetic field has to be solved since its gradient changes as the concentration of the magnetic particles changes. Finally, we have compared the simulation result with the experimental one to verify the validity of the simulation method.

\section{NUMERICAL SimUlation METHOD}

\section{A. Magnetic Field Simulation}

The magnetic force $\boldsymbol{F}_{\text {mag }}$ on a fine magnetic particle is given by [13], [14]

$$
\boldsymbol{F}_{\text {mag }}=\mu_{0}(\boldsymbol{M} \cdot \nabla) \boldsymbol{H}
$$

where $\mu_{0}, \boldsymbol{M}$ and $\boldsymbol{H}$ are the permeability in the free space, the magnetization of the magnetic particle and the magnetic field, respectively. The magnetic field gradient, which is generated by fine ferromagnetic wires on the channel wall in this study, generates the magnetic force. The strength of the magnetic force depends on the susceptibility of the magnetic particle and the magnetic field gradient.

The magnetic field is solved by the magnetic moment method [15], [16]. The magnetization $\boldsymbol{M}$ is obtained from 


$$
\begin{aligned}
& -\frac{1}{4 \pi} \int_{V}\left(\nabla^{\prime} \cdot \boldsymbol{M}\left(\boldsymbol{r}^{\prime}\right)\right) \frac{\boldsymbol{r}-\boldsymbol{r}^{\prime}}{\left|\boldsymbol{r}-\boldsymbol{r}^{\prime}\right|^{3}} \mathrm{~d} V^{\prime}+\frac{1}{4 \pi} \int_{S}\left(\boldsymbol{M}\left(\boldsymbol{r}^{\prime}\right) \cdot \boldsymbol{n}\right) \frac{\boldsymbol{r}-\boldsymbol{r}^{\prime}}{\left|\boldsymbol{r}-\boldsymbol{r}^{\prime}\right|^{3}} \mathrm{~d} S^{\prime} \\
& -\frac{1}{\chi_{m}(\boldsymbol{r})}=-\boldsymbol{B}_{F}(\boldsymbol{r})
\end{aligned}
$$

where $\boldsymbol{r}$ and $\boldsymbol{r}$ are the position vector, $\chi_{m}$ the susceptibility, and $\boldsymbol{B}_{F}$ the magnetic field generated by the superconducting magnet, respectively. The magnetic field $\boldsymbol{H}$ is obtained substituting $\boldsymbol{M}$ for

$$
\boldsymbol{H}=\frac{1}{\mu_{0} \chi_{m}} \boldsymbol{M}
$$

In the magnetic field analysis, it is assumed that the magnetization $\boldsymbol{M}$ of the particle is parallel to the magnetic field $\boldsymbol{H}$ and the angular momentum of the particles doesn't exist. On this study, the magnetic moment method is adequate since the scale of the superconducting magnet is much different from that of the magnetic fluid. That is, the integral method is more accurate than the domain method, such as finite element method.

In the magnetic moment method, the tetrahedral element is employed, and the magnetization $\boldsymbol{M}$ is constant in element. The magnetic field $\boldsymbol{H}$ at the vertices of tetrahedron is also obtained from (2). The magnetic force of (1) is given by the obtained magnetization $\boldsymbol{M}$ in an element and the magnetic field $\boldsymbol{H}$ at its vertices.

The magnetization of the magnetic particle is given by [14]

$$
\boldsymbol{M}=\phi M_{S} L\left(\frac{\mu_{0} m|\boldsymbol{H}|}{k T}\right) \frac{\boldsymbol{H}}{|\boldsymbol{H}|}
$$

where $\phi$ is the concentration of the magnetic particles, $M_{S}$ the saturation magnetization, $L$ the Langevin function, $k$ the Boltzmann constant, $T$ the absolute temperature, and $m=V_{P} M_{S}$, where $V_{P}$ is the volume of a magnetic particle. The magnetic susceptibility is dependent on the size of magnetic particle, but, in this study, the magnetic field is so strong that the Langevin function $L$ is almost equal to 1.0. That is, the magnetization of the magnetic fluid is proportional to the concentration of the magnetic particles.

\section{B. Navier-Stokes Simulation}

The fluid velocity $\boldsymbol{v}$ in the flow channel is obtained by solving the Navier-Stokes equation [14], [17],

$$
\rho \frac{\partial \boldsymbol{v}}{\partial t}+\rho \boldsymbol{v} \cdot \nabla \boldsymbol{v}=-\nabla p^{*}+\mu_{0}(\boldsymbol{M} \cdot \nabla) \boldsymbol{H}+\eta \nabla^{2} \boldsymbol{v}
$$

where $\rho, p^{*}$ and $\eta$ are the fluid density, the composite pressure and the coefficient of viscosity, respectively. The angular momentum of the particles is ignored. The second term in right side, the magnetic force, is computed from the magnetic field analysis, mentioned above. The Navier-Stokes equation is solved by the stabilized finite element, that is the streamline
upwind/Petrov-Galerkin and Pressure stabilizing/PetrovGalerkin method, a popular in the field of dynamic fluid simulation [17]. The common tetrahedral element is employed with the magnetic field analysis, in this study.

\section{Control Volume Simulation}

The governing equation of the control volume is

$$
\frac{\partial}{\partial t}(\rho \phi)+\nabla \cdot(\rho \boldsymbol{v} \phi)=\nabla \cdot(\Gamma \nabla \phi)+S
$$

where $\Gamma$ and $S$ are the scalar diffusion constant and the scalar source term, respectively. The fluid velocity $v$ is obtained from the Navier-Stokes equation. The concentration $\phi$ of the magnetic particles is given by solving (6), taking into account the diffusion of the magnetic particles in the fluid. The concentration $\phi$ is fed back to computing the magnetization of the magnetic particles in (4). The ordinary finite element method with the common tetrahedral elements is used, in this study.

\section{Simulation Algorithm}

For accurately simulating the fluid flow with the magnetic particles in the magnetic column, the Navier-Stokes, the control volume and the magnetic field equations are solved simultaneously. Fig. 1 shows the flowchart of the simulation combining the fluid dynamics and the magnetics. At first, the magnetization of the magnetic particles and the magnetic field is obtained by solving (2), (3) and (4). Substituting the obtained magnetization and magnetic field into (5), the fluid velocity and the composite pressure are computed. Then, the concentration of the magnetic particles is computed by solving (6) with the obtained fluid velocity. A series of simulations are repeated till the analysis time ends.

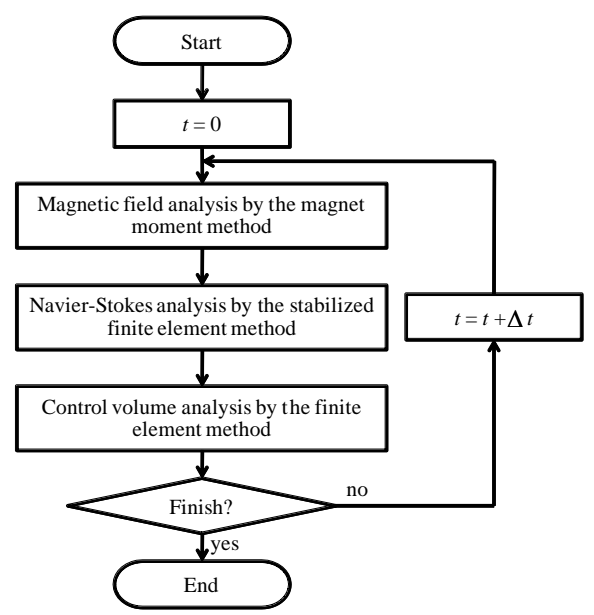

Fig. 1. The flowchart of the developed ferrohydrodynamics and magnetic field simulation. The magnetic field, the Navier-Stokes and the control volume analyses are done in each time-step.

\section{EXPERIMENT}

In order to confirm the validity of the simulation method mentioned above, the simulation results were compared to 
experimental ones. The detail of the experimental setup was reported in [6] and [7]. Fig 2 shows the magnetic column in this study. The fine ferromagnetic wires of $0.2 \mathrm{~mm}$ width are alternately located on the upper and lower walls of the flow channel inside the magnetic column, which is made of $7 \%$ Nickel and $93 \%$ Cobalt. The body of the magnetic column is made of non-magnetic stainless steel. The fluid with the magnetic particle flows through the flow channel of $10 \mathrm{~mm}$ width and $0.17 \mathrm{~mm}$ height. The superconducting magnet applies 2.0 T magnetic field to the column.

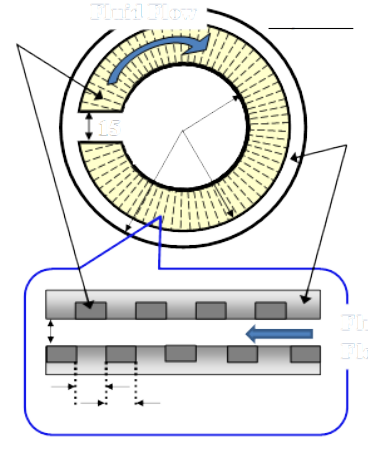

Fig. 2. The schematic view of the magnetic column and the flow channel of $0.17 \mathrm{~mm}$ height and $10 \mathrm{~mm}$ width. The fine ferromagnetic wires of $0.2 \mathrm{~mm}$ width are vertically and alternately arranged against the flow direction with $0.2 \mathrm{~mm}$ apart.

\section{Simulation Results}

\section{A. Simulation Conditions}

The flow channel is too long to simulate within a realistic computation time, so the $1 / 10$ of average length of the flow channel is modeled. Table I shows the numbers of nodes and elements. The time step is $0.1 \mathrm{~ms}$. The magnetic particles are collectively taken into account in each element, since it was assumed that the magnetization of the magnetic particle is parallel to the magnetic field, and the angular momentum of the particles doesn't exist.

TABLE I

NUMBERS OF NODES AND ELEMENTS

\begin{tabular}{lrr}
\hline \hline & \multicolumn{1}{c}{ Nodes } & \multicolumn{1}{c}{ Elements } \\
\hline Flow Channel & 33,936 & 180,000 \\
Ferromagnetic Wires & 2,592 & 5,760 \\
\hline \hline
\end{tabular}

For ferrohydrodynamics analysis, only the mesh of the flow channel is used. For magnetic field analysis, the meshes of both the flow channel and ferromagnetic wires are used.

\section{B. Comparison with the Experiment}

The simulation of the magnetic fluid flow thorough the developed magnetic column was performed, and its result was compared with the experimental one. Magnetic particles are used in the experiment $\mathrm{Fe}_{3} \mathrm{O}_{4}$ particles of $300 \mathrm{~nm}$ average diameter. On the experiment, pure water flows into the magnetic column with $5.0 \mathrm{ml} / \mathrm{min}$ velocity. Before flowing into the magnetic column, the magnetic particles are injected into the pure water for $1.0 \mathrm{~s}$.

Fig. 3 shows the time-varying concentration of the magnetic particles at the outlet of the magnetic column. The waveforms of the numerical and measured normalized intensity are comparable.

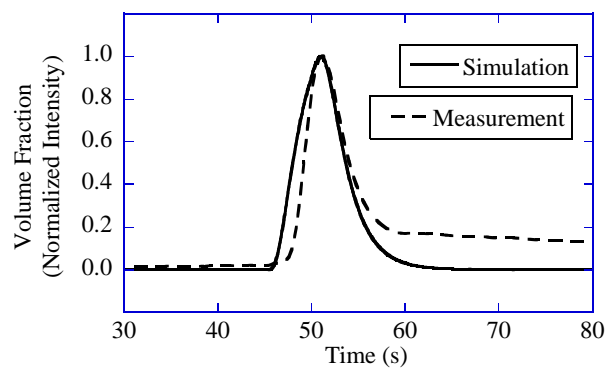

Fig. 3. The simulated and measured time-variation of the normalized intensity of the magnetic particle in the outlet fluid. The flow velocity is $5.0 \mathrm{ml} / \mathrm{min}$.

\section{Simulation Result of the Proposed Method}

Fig. 4 shows the concentration of the flow simulation on the horizontal cross-section of the flow channel. The concentration distribution in the direction of width is almost uniform. Fig. 5 (a) and (b) show the concentration and the flow of the magnetic fluid on the vertical cross-section, respectively. The meandering flow is observed only in front of the collective magnetic particles. The flow is almost straight after the front. The collective magnetic particles with susceptibility are filled inside the flow channel, so that the magnetic field gradient weakens.

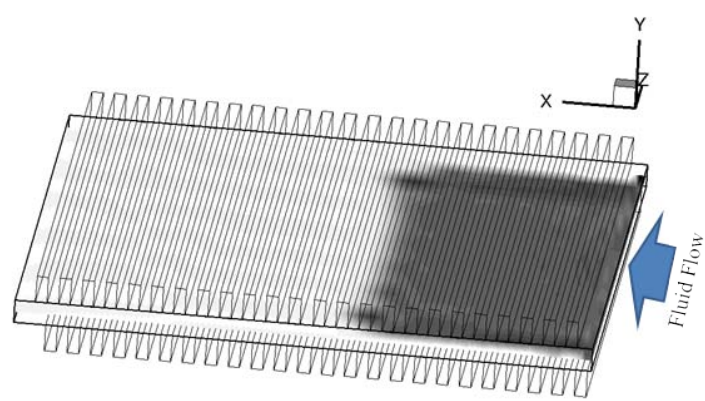

Fig. 4. An example of the concentration of the magnetic particles on the horizontal cross-section of the flow channel. The ferromagnetic wires are also depicted.

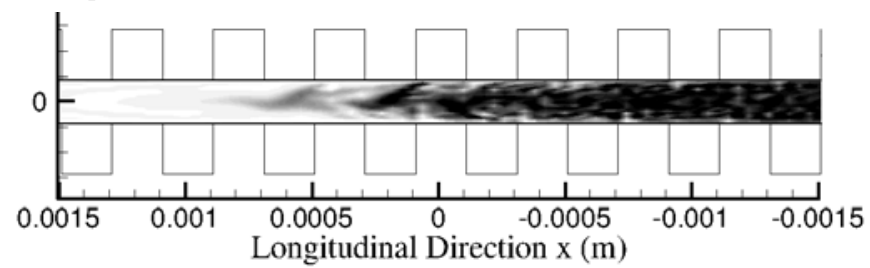

(a)

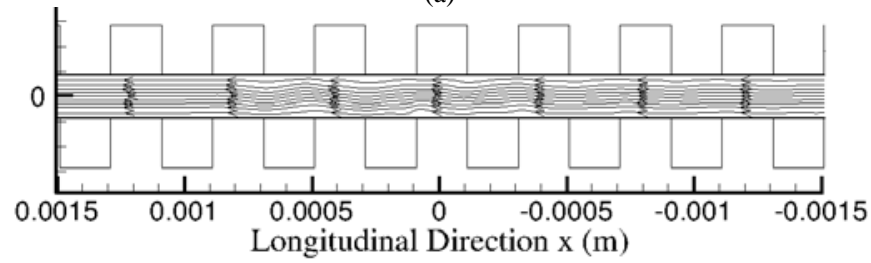

(b)

Fig. 5. (a) Concentration of the magnetic particles on the vertical crosssection of the flow channel and (b) the fluid flow. These results are obtained by the newly developed simulation method. The meandering flow is observed only in the front of the collective magnetic particles. 


\section{Comparison with the Previous Simulation Method}

To verify the effectiveness of the proposed method, its result is compared with that of the previously proposed simulation method [18], in which the magnetic field is analyzed only once before simulating the time-varying fluid flow. Fig. 6 (a) and (b) shows the magnetic particle concentration and the flow obtained by the previous simulation method, respectively. The largely meandering flow continues after the front of the collective magnetic particles, since the magnetic field changing by the magnetic particles isn't taken into account. The phenomenon of the previous simulation method is obviously different from that of the proposed simulation method.

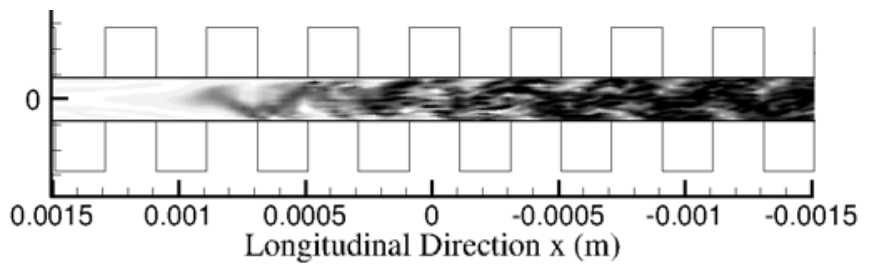

(a)

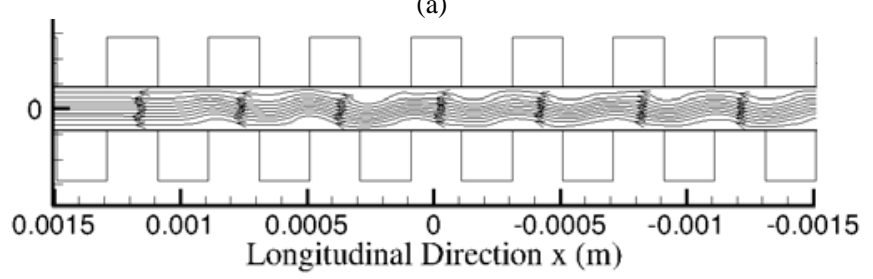

(b)

Fig. 6. (a) Concentration of the magnetic particles on the vertical crosssection of the flow channel and (b) the fluid flow. Both are obtained by the previous simulation method [18]. The largely meandering flow continues after the front of the collective magnetic particles.

\section{CONCLUSION}

We have developed the magnetic fluid simulation code for analyzing magnetic particle separation systems. Navier-Stokes, control volume and magnetic field equations are taken into account simultaneously in order to enhance the simulation accuracy.

The result of the developed simulation method was compared with the experimental one, with fairly good agreement. Then, the results of the previous and the newly developed simulation method were also compared. A wrong meandering flow was observed in the result of the previous simulation method. On the other hand, the newly developed simulation method was more accurate.

The developed code needs to be optimized since simulation time of the magnetic field analysis was 10 times that one of fluiddynamics analysis.

\section{ACKNOWLEDGMENT}

This work was supported in part by a Grant for Young Scientists (B) of the Ministry of Education, Culture, Sports, Science and Technology.

\section{REFERENCES}

[1] S. Nishijima and S. Takeda, "Superconducting High Gradient Magnetic Separation for Purification of Wastewater From Paper Factory," IEEE Transactions on Applied Superconductivity, vol. 16, no. 2, pp. 11421145, Jun. 2006.

[2] S. Takeda, and S. Nishijima, "Development of Magnetic Separation of Water-Soluble Materials Using Superconducting Magnet," IEEE Transactions on Applied Superconductivity, vol. 17, no. 2, pp. 21782180, Jun. 2007.

[3] F. Mishima, T. Terada, Y. Akiyama, Y. Izumi, H. Okazaki, and S. Nishijima, "Research and Development of Superconducting Magnetic Separation System for Powdered Products," IEEE Transactions on Applied Superconductivity, vol. 18, no. 2, pp. 824-827, Jun. 2008.

[4] H. Ueda, K. Agatsuma, K. Kajikawa, M. Furuse, S. Fuchino, and A. Ishiyama, "Design and Test of Filter of High Gradient Magnetic Separation System for Trapping Immunoglobulin in Serum," IEEE Transactions on Applied Superconductivity, vol. 19, no. 3, pp. 21572161, Jun. 2009.

[5] Y. Nakai, F. Mishima, Y. Akiyama, and S. Nishijima, "Development of Magnetic Separation System for Powder Separation," to be published in IEEE Transactions on Applied Superconductivity, 2010.

[6] S. B. Kim, C. Nakata, S. Murase, H. Okada, and T. Ohara, "Development of magnetic chromatograph system for magnetic particle and ion separation with superconducting magnet," Physica C, vols. 463465, pp. 1306-1310, 2007.

[7] S. B. Kim, Y. Okimoto, S. Murase, S. Noguchi, and H. Okada, "Characteristics of Magnetic Separation for Magnetic Particle and Ion by Magnetic Chromatography With Novel Magnetic Column,” IEEE Transactions on Applied Superconductivity, vol. 19 no. 3, pp. 2152-2156, Jun. 2009.

[8] S. Fukui, H. Nakajima, A. Ozone, M. Hayatsu, M. Yamaguchi, T. Sato, H. Imaizumi, S. Nishijima, and T. Watanabe, "Study on Open Gradient Magnetic Separation Using Multiple Magnetic Field Sources,” IEEE Transactions on Applied Superconductivity, vol. 12, no. 1, pp. 959-962, Mar. 2002.

[9] S. Fukui, M. Takahashi, T. Fujita, M. Yamaguchi, T. Sato, H. Imaizumi, M. Oizumi, S. Nishijima, and T. Watanabe, "Analytical Study on Open Gradient Magnetic Separation Using Quadrupole Magnetic Field,” IEEE Transactions on Applied Superconductivity, vol. 14, no. 2, pp. 15721575, Jun. 2004.

[10] M. Takahashi, S. Fukui, Y. Takahashi, R. Abe, J. Ogawa, M. Yamaguchi, T. Sato, H. Imaizumi, and T. Ohara, "Numerical Study on Magnetic Chromatography Using Quadrupole Magnetic Field,” IEEE Transactions on Applied Superconductivity, vol. 16, no. 2, pp. 11161119, Jun. 2006.

[11] M. R. Smolkin and R. D. Smolkin, "Calculation and Analysis of the Magnetic Force Acting on a Particle in the Magnetic Field of Separator. Analysis of the Equations Used in the Magnetic Methods of Separation," IEEE Transactions on Magnetics, vol. 42, no. 11, pp. 3682-3693, Nov. 2006.

[12] R. Mehasni, M. Feliachi, and M. E. H. Latreche, "Effect of the Magnetic Dipole-Dipole Interaction on the Capture Efficiency in Open Gradient Magnetic Separation,” IEEE Transactions on Magnetics, vol. 42, no. 8, pp. 3488-3493, Aug. 2007.

[13] K. C. Warnke, "Finite-Element Modeling of the Separation of Magnetic Microparticles in Fluid," IEEE Transactions on Magnetics, vol. 39, no. 3, pp. 1771-1777, May 2003.

[14] R. E. Rosensweig, Ferrohydrodynamics, Dover Publications, Inc., 1997.

[15] Y. Takahashi, S. Wakao, and A. Kameari, "Large-scale and Highly accurate Magnetic Field Analysis of Magnetic Shield," Journal of Applied Physics, vol. 99, no. 8, H904, 2006.

[16] Y. Takahashi, C. Matsumoto, and S. Wakao, "Large-scale and Fast Nonlinear Magnetostatic Field Analysis by the Magnetic Moment Method with Adaptive Cross Approximation,” IEEE Transactions on Magnetics, vol. 43, no. 4, pp. 1277-1280, 2007.

[17] J. H. Ferziger and M. Peric, Computational Methods for Fluid Dynamics, Springer-Verlag, Berlin Heidelberg, 1996.

[18] S. Noguchi, S. B. Kim, R. Iwamoto, K. Kataoka, and H. Okada, "Development of Numerical Analysis Method for Magnetic Particle and Ion with Magnetic Chromatography," presented at 21st International Conference on Magnet Technology, Heifei, 2009. 